Pacific Journal of Mathematics

Sours 


\title{
REPRESENTATIONS OF DIRECT PRODUCTS OF FINITE GROUPS
}

\author{
BuRTON FEIN
}

Let $G$ be a finite group and $K$ an arbitrary field. We denote by $K(G)$ the group algebra of $G$ over $K$. Let $G$ be the direct product of finite groups $G_{1}$ and $G_{2}, G=G_{1} \times G_{2}$, and let $M_{i}$ be an irreducible $K\left(G_{i}\right)$-module, $i=1,2 . \quad$ In this paper we study the structure of $M_{1}, M_{2}$, the outer tensor product of $M_{1}$ and $M_{2}$.

While $M_{1}, M_{2}$ is not necessarily an irreducible $K(G)$ module, we prove below that it is completely reducible and give criteria for it to be irreducible. These results are applied to the question of whether the tensor product of division algebras of a type arising from group representation theory is a division algebra.

We call a division algebra $D$ over $K K$-derivable if $D \cong$ $\operatorname{Hom}_{K(G)}(M, M)$ for some finite group $G$ and irreducible $K(G)$ module $M$. If $B(K)$ is the Brauer group of $K$, the set $B_{0}(K)$ of classes of central simple $K$-algebras having division algebra components which are $K$-derivable forms a subgroup of $B(K)$. We show also that $B_{0}(K)$ has infinite index in $B(K)$ if $K$ is an algebraic number field which is not an abelian extension of the rationals.

All $K(G)$-modules considered are assumed to be unitary finite dimensional left $K(G)$-modules. If $M_{i}$ is a $K\left(G_{i}\right)$-module, $i=1$, 2, the outer tensor product $M_{1} \# M_{2}$ of $M_{1}$ and $M_{2}$ is the $K(G)$-module whose underlying space is $M_{1} \otimes_{K} M_{2}$ and where $\left(g_{1}, g_{2}\right) \in G$ acts on $M_{1} \otimes_{K} M_{2}$ by

$\left(g_{1}, g_{2}\right) \sum m_{i} \otimes m_{i}^{\prime}=\sum g_{1} m_{i} \otimes g_{2} m_{i}^{\prime}, m_{i} \in M_{1}, m_{i}^{\prime} \in M_{2}, g_{j} \in G_{j}, j=1,2$.

It will be necessary to refer to the theory of the Schur index of absolutely irreducible representations of finite groups. In $\S 1$ we present a treatment of this theory where the relevant theorems are proved for arbitrary fields. This treatment is included in the author's doctoral dissertation supervised by Professor Charles W. Curtis at the University of Oregon. During the preparation of this paper the author held a National Science Foundation Graduate Fellowship.

1. The Schur index. The method used in [3, § 70] to prove the relevant theorems about the Schur index for fields of characteristic zero does not seem to generalize to arbitrary fields. In that treatment attention is focused on the enveloping algebra of the representations rather than on the representations themselves. We work directly with modules 
over group algebras. After Theorem 1.1 has been proved, the methods of $[3, \S 70]$ can be generalized to arbitrary fields. However, this approach seems to be unnecessarily long and complicated and we have chosen to present a unified treatment independent of these methods. For the convenience of the reader we have included several short arguments that are similar to ones appearing in [3].

Before we can state our main results we need to introduce some terminology. We refer the reader to [3] for the relevant theory.

Let $G$ be a finite group. A field $E$ is a splitting field for $G$ if every irreducible $E(G)$-module is absolutely irreducible. Let $K$ be a field. By Theorem 69.11 of [3] there is a finite normal separable extension $E$ of $K$ which is a splitting field for $G$. For if $K$ has characteristic $p$, there is a finite field $F$ of characteristic $p$ which is a splitting field for $G$. Since $F$ is an extension of its prime field by roots of unity, a composite $E=F \cdot K$ of $F$ and $K$ is a splitting field of the desired type. We shall assume throughout this section that $E$ is a normal separable extension of $K$ which is splitting field for $G$. $K$ will be assumed to be an arbitrary field.

We denote the Galois group of $E$ over $K$ by $\mathscr{G}(E \mid K)$. Let $N$ be an $E(G)$-module with basis $m_{1}, \cdots, m_{n}$ over $E$, and let the action of $G$ on $N$ be given by $g m_{i}=\sum_{j} a_{i j}(g) m_{j}, g \in G, a_{i j}(g) \in E$. Let $V$ be an $n$ dimensional vector space over $K$ with basis $v_{1}, \cdots, v_{n}$ and let $\sigma \in \mathscr{G}(E \mid K)$. Under the action $g v_{i}=\sum_{j} \sigma\left(a_{i j}(g)\right) v_{j}, g \in G, V$ becomes an $E(G)$-module which we denote by $\sigma N$. $\sigma N$ is called a conjugate module of $N$. If $\chi$ is the character of $N$, then we denote by $\sigma \chi$ the character of $\sigma N$, where $(\sigma \chi)(g)=\sigma(\chi(g)), g \in G . \quad \sigma$ and $\tau$ will always denote elements of $\mathscr{G}(E \mid K)$ while $\chi$ and $\psi$ will always be characters of modules over group algebras.

Let $N$ be an irreducible $E(G)$-module and let $E^{*}$ denote an algebraic closure of $E$. All fields considered will be assumed to be subfields of $E^{*} . \quad N^{*}=N \bigotimes_{E} E^{*}$ is an irreducible $E^{*}(G)$-module. $\quad N^{*}$ is said to be realizable in a subfield $J$ of $E^{*}$ if there is a $J(G)$-module $V$ such that $V \otimes_{J} E^{*} \cong N^{*}$. Let $\chi$ be the character of $N, \chi^{*}$ the character of $N^{*}$. Then $\chi^{*}(g)=\chi(g)$ for all $g \in G$. We denote by $K(\chi)$ the field generated over $K$ by the values $\chi(g), g \in G$. The Schur index $m_{K}(N)$ of $N$ over $K$ is the minimum value of $(J: K(\chi))$, the degree of $J$ over $K(\chi)$, taken over all fields $J$ in which $N^{*}$ is realizable, where $K(\chi) \subset J \subset E^{*}$. In general, there will not exist a subfield $J$ of $E$ in which $N$ is realizable and such that $(J: K(\chi))=m_{K}(N)$ [2].

Let $M$ be an irreducible $K(G)$-module. $\quad M$ is isomorphic to a minimal left ideal of a simple component $A$ of $K(G) / \operatorname{rad} K(G)$ [3, Th. 25.10]. $A$ is isomorphic to a complete matrix $\operatorname{ring}(D)_{n}, D$ a division algebra with center $L, L \supset K$, and $D \cong \operatorname{Hom}_{K(G)}(M, M)$ [3, Th. 26.8]. The index $m(D)$ of $D$ is $(F: L)$ where $F$ is any maximal subfield of 
$D$ [3, Th. 68.6]. We shall let $r M$ denote the direct sum of $r$ copies of $M$, where $r$ is a natural number. We set $M^{E}=M \otimes_{K} E$. $\quad N$ will be assumed to be an irreducible $E(G)$-module which is a composition factor of $M^{E}$. $\chi$ will be the character of $N$. Since $A$ is associative, $A$ may be viewed as an $L$-algebra. We denote this algebra by ${ }_{L} A$. $A$ will denote ${ }_{K} A$. We shall maintain the above context throughout this entire section.

THEOREM 1.1. The center $L$ of $D$ is $K(\chi) . A \otimes_{K} K(\chi)$ is isomorphic to a direct sum of $t$ copies of ${ }_{K(\chi)} A$, where $t=(K(\chi): K)$.

We begin with a lemma which is essentially proved in [3, Th. $70.15]$.

Lemma 1.2: $M^{E} \cong k\left(\sigma_{1} N \oplus \cdots \oplus \sigma_{t} N\right)$ where the $\sigma_{i} \in \mathscr{G}(E \mid K)$, $\sigma_{1}=1, k$ is a natural number, the $\left\{\sigma_{i} N\right\}$ form a complete set of nonisomorphic conjugates of the irreducible $E(G)$-module $N$, and $t=$ $(K(\chi): K)$.

Proof. $M^{E}$ is a completely reducible and $E \otimes_{K}(K(G) / \operatorname{rad} K(G)) \cong$ $E(G) / \operatorname{rad} E(G)$ [3, Ths. 69.9, 69.10]. $A^{E}$ is a component (not necessarily simple) of $E(G) / \mathrm{rad} E(G)$. Since $A^{E}$ is semi-simple [3, Th. 69.4] we have $A^{E}=C \cong C e_{1} \oplus \cdots \oplus C e_{t}$ where the $e_{i}$ are primitive orthogonal central idempotents of $C$. For any $\sigma \in \mathscr{G}(E \mid K)$ we define a $K$ automorphism of $A^{E}$ by $\sigma\left(\sum a_{j} \otimes f_{j}\right)=\sum a_{j} \otimes \sigma f_{j}, a_{j} \in A, f_{j} \in E . \quad \sigma\left(f_{i}\right)$ is again a primitive central idempotent of $C$ and so coincides with some $f_{j}, 1 \leqq j \leqq t$. If $f_{1}, \cdots, f_{r}$ are the different conjugates $\sigma\left(f_{1}\right)$ of $f_{1}$ then $f=f_{1}+\cdots+f_{r}$ is a central idempotent of $A$. Since $A$ is simple, $r=t$. Let $f_{1}=\sum f_{1 j}$ be the decomposition of $f_{1}$ into primitive idempotents. $C f_{1 j} \cong N$ for some irreducible $E(G)$-module $N$ and $C \sigma\left(f_{1 j}\right) \cong \sigma N$. Since $C \cong C f_{1} \oplus C \sigma_{2}\left(f_{1}\right) \oplus \cdots \oplus C \sigma_{t}\left(f_{1}\right)$, we see that $N, \sigma_{2} N, \cdots, \sigma_{t} N$ are the distinct $E(G)$-components of $M^{E}$ and that the $\left\{\sigma_{i} N\right\}$ form a complete set of nonisomorphic conjugates of $N$. This proves that $M^{E} \cong d(1) N \oplus d(2) \sigma_{2} N \oplus \cdots \oplus d(t) \sigma_{t} N$, where the $d(i)$ are natural numbers. Since $\sigma M^{E} \cong M^{E}$ for all $\sigma \in \mathscr{G}(E \mid K)$,

$$
d(1) N \oplus \cdots \oplus d(t) \sigma_{t} N \cong d(1) \sigma N \oplus d(2) \sigma \sigma_{2} N \oplus \cdots \oplus d(t) \sigma \sigma_{t} .
$$

By the Krull-Schmidt Theorem $d(1)=d(2)=\cdots=d(t)$. It only remains to prove that $t=(K(\chi): K)$. Let $\mathscr{C}=\{\sigma \in \mathscr{G}(E \mid K) \mid \sigma N \cong N\}$. From Galois Theory $t=[\mathscr{G}(E \mid K): \mathscr{C}]$. But $\sigma N \cong N$ if and only if $\sigma \chi=\chi$, where $\chi$ is the character of $N$. Therefore

$$
\mathscr{H}=\{\sigma \in \mathscr{G}(E \mid K) \mid \sigma \chi=\chi\}
$$


and so $t=(K(\chi): K)$.

Let $h$ be the exponent of $G$. For $g \in G, \chi(g)$ is a sum of $h$-th roots of unity. Therefore $K(\chi) \subset K(\sqrt[h]{1})$ and since $\mathscr{C}(K(\sqrt[h]{1}) \mid K)$ is abelian $K(\chi)$ is a normal separable extension of $K$. If $\sigma \in \mathscr{G}(E \mid K)$, then $K(\sigma \chi)=K(\chi)$.

Proof of Theorem 1.1. Let $A \otimes_{K} K(\chi) \cong B_{1} \oplus \cdots \oplus B_{s}$, the $B_{i}$ simple $K(\chi)$-algebras. If the irreducible $K(\chi)(G)$-module $U$ is isomorphic to a minimal left ideal of $B_{1}$, then $U^{E} \cong r\left(\sigma_{i} N \oplus \cdots\right)$ by Lemma 1.2. However, since $K(\sigma \chi)=K(\chi)$ for all $\sigma \in \mathscr{G}(E \mid K)$, it follows that $U^{E} \cong r(\sigma N)$ for some $\sigma \in \mathscr{G}(E \mid K)$. Since $A^{E}$ has $t$ distinct nonisomorphic simple components we have $s \leqq t$ and $B_{i} \not B_{j}$ for all $i, j$. Therefore $s=t$ and each $B_{i} \otimes_{K(x)} E$ is simple with center $E$ [3, Th. 29.13]. If $F_{i}$ is the center of $B_{i}$, then the centroid of $B_{i} \otimes_{K(x)} E$ is $F_{i} \otimes_{K(x)} E$ [7, Th. 1, p. 114]. Counting dimensions we see that $F_{i} \otimes_{K(\chi)} E \cong E$ if and only if $F_{i}=K(\chi)$. Therefore the centroid of $A \otimes_{K} K(\chi)$ is isomorphic to a direct sum of $t$ copies of $K(\chi)$. The center of $D$ is $L$. Then the centroid of $A \otimes_{K} K(\chi)$ is $L \otimes_{K} K(\chi)$ and so $L \otimes_{K} K(\chi)$ is a direct sum of $t$ copies of $K(\chi), t=(K(\chi): K)$. If $I$ is a maximal ideal of $L \otimes_{K} K(\chi)$, then $\left(L \otimes_{K} K(\chi)\right) / I \cong K(\chi)$ and so every composite of $K(\chi)$ and $L$ over $K$ is isomorphic to $K(\chi)$ [8, p. 84, Th. 21]. Therefore $K(\chi)=L$. But then $A$ is an algebra over $K(\chi)$ and we have

$$
\begin{aligned}
A \otimes_{K} L \cong\left({ }_{L} A \otimes_{L} L\right) \otimes_{K} L \cong{ }_{L} A \otimes_{L}\left(L \otimes_{K} L\right) \\
\quad \cong_{L} A \otimes_{L}(L \oplus \cdots \oplus L) \cong{ }_{L} A \otimes_{L} L \oplus \cdots \oplus_{L} A \otimes_{L} L \\
\quad \cong{ }_{L} A \oplus \cdots \oplus{ }_{L} A .
\end{aligned}
$$

Since $L$ is normal over $K, L \otimes_{K} L$ is a direct sum of $t$ copies of $L$, $t=(L: K)[8$, p. 87].

It will always be clear from the context whether we are viewing $A$ as a $K$-algebra or as an $L$-algebra. We shall, therefore, not continue to distinguish between these algebras but shall simply write $A$ for both ${ }_{K} A$ and ${ }_{L} A$. We recall that a finite extension $F$ of $L$ is a splitting field for $D\left(A=(D)_{n}\right)$ if $D \otimes_{L} F=(F)_{s}$ for some integer $s$.

LEMMA 1.3. Let $K$ be a perfect field and $F$ a finite extension of $L$. Then $F$ is a splitting field for $D$ if and only if $N^{*}$ is realizable in $F, N^{*}=N \otimes_{*} E^{*}$.

Proof. Since $F$ is a separable extension of $L$.

$$
F \otimes_{L} L(G) / \operatorname{rad} L(G) \cong F(G) / \operatorname{rad} F(G)
$$


[3, Th. 69.10]. Let $U$ be an $L(G)$-module so that $U \otimes_{L} E^{*} \cong r N^{*}$, $r$ a natural number, and such that $U$ is isomorphic to a minimal left ideal of $A$ (the existence of such a $U$ was proved in the proof of Theorem 1.2). $A \otimes_{L} F$ is a simple component of $F(G) / \operatorname{rad} F(G)$. Let $V$ be a minimal left ideal of $A \otimes_{L} F$. Then $\operatorname{Hom}_{F(\theta)}(V, V) \cong D^{\prime}$ where $A \otimes_{L} F=\left(D^{\prime}\right)_{v} . \quad N^{*}$ is realizable in $F$ if and only if $D^{\prime}=F$ [3, Th. 29.13]. Since $F$ is a splitting field for $D$ if and only if $A \otimes_{L} F=(F)_{v}$ we are done.

THEOREM 1.4. (a) $M^{E} \cong m_{K}(N)\left(N \oplus \sigma_{2} N \oplus \cdots \oplus \sigma_{t} N\right)$ where the $\sigma_{i} \in \mathscr{G}(E \mid K)$, the $\left\{\sigma_{i} N\right\}$ form a complete set of nonisomorphic conjugates of the irreducible $E(G)$-module $N$, and $t=(K(\chi): K)$.

(b) $m_{K}(N)=m(D)$.

(c) If $K$ has prime characteristic, then $m_{K}(N)=1$, i.e. $\operatorname{Hom}_{K(G)}(M, M)$ is commutative.

(d) $m_{K}(N)$ divides the dimension $(N: E)$ of $N$ over $E$.

(e) For any finite algebraic extension $J$ of $K$ in which $N^{*}$ is realizable, $m_{K}(N)$ divides $(J: K(\chi))$.

Proof. We have $A \otimes_{K} E=C \cong C e_{1} \oplus \cdots \otimes C \sigma_{t}\left(e_{1}\right)$. Since $E$ is a splitting field for $G, C e_{1} \cong C \sigma_{i}\left(e_{1}\right) \cong(E)_{r}$

$$
\begin{aligned}
& \left(A \otimes_{K} E: E\right)=t r^{2}=(A: K)=\left((D)_{n}: K\right)=n^{2}(D: K) \\
& \quad=n^{2}(D: L)(L: K)=n^{2} t[m(D)]^{2} .
\end{aligned}
$$

Therefore $r=n \cdot m(D) . \quad M$ is isomorphic to a minimal left ideal $I$ of $A$. Since $A=(D)_{n}, A$ is isomorphic to a direct sum of $n$ copies of $I$. Set $m=m(D)$. Then $A \otimes_{K} E$ is isomorphic to a direct sum of $t$ copies of $(E)_{m n}$ so $A \bigotimes_{K} E$ is a direct sum of $t m n$ minimal left ideals. $\sigma_{j} N$ is isomorphic to a minimal left ideal $I_{j}$ of $A \bigotimes_{K} E, j=1, \cdots, t$, $\sigma_{1}=1$. Since $M \cong I$, the $\left\{I_{j}\right\}$ appear with equal multiplicity in $I \otimes_{K} E$. By Lemma $1.2 k=m(D)$ and $M^{E} \cong m(D)\left(N \oplus \sigma_{2} N \oplus \cdots \oplus \sigma_{t} N\right)$. The rest of the proof is divided into two parts.

Case 1. $K$ is perfect. Let $V$ be a maximal subfield of $D . \quad V$ is a splitting field for $D$ of minimal $K$-dimension. By Lemma $1.3 \mathrm{~N}^{*}$ is realizable in $V$. Therefore $m_{K}(N) \geqq(V: L)=m(D)$. Conversely, if $N^{*}$ is realizable in a finite extension $F$ of $L$ then $F$ is a splitting field for $D$. Hence $m_{K}(N) \leqq m(D)$. This proves (a) and (b) when $K$ is perfect. Let $K$ now have characteristic zero. We have seen that $N$ is isomorphic to a minimal left ideal of $(E)_{n m}$. Then $\operatorname{Hom}_{E}(N, N) \cong$ $(E)_{m n}$ so $(N: E)=n m=n \cdot m_{K}(N)$. If $N^{*}$ is realizable in a finite algebraic extension $J$ of $K$, then $J$ is a splitting field for $D$ by Lemma 1.3. $m(D)=m_{K}(N)$ divides $(J: K(\chi))$ by [3, Th. 68.7]. This proves 
(d) and (e) for $K$ of characteristic zero.

Case 2. $K$ has characteristic $p, p>0$. Assume first of all that $K$ is finite. Then $D$ is a finite skewfield and hence a field, $D=K(\chi)$ [3, Th. 68.9]. Since $K$ is perfect and $K(\chi)$ is a splitting field for $D$, $N^{*}$ is realizable in $K(\chi)$. Therefore $m_{K}(N)=1$ by Case 1 . We have $m(D)=1$ also. We now assume that $K$ is infinite. Let $F=Z_{p}(\chi$, $\left.\sigma_{2} \chi, \cdots, \sigma_{t} \chi\right)$ where $Z_{p}$ is the prime field and the $\left\{\sigma_{i} \chi\right\}$ are the characters of the $\left\{\sigma_{i} N\right\} . \quad F$ is a finite field so the $\left\{\sigma_{i} N\right\}$ are all realizable in $F$, say $V_{i} \bigotimes_{F} E \cong \sigma_{i} N, i=1, \cdots, t, \sigma_{1}=1$. The $V_{i}$ are irreducible $F(G)$-modules. Let $W=V_{1} \oplus \cdots \oplus V_{t}$. The character of $W$ lies in $K \cap F=R$. Since $F \otimes_{R} R(G) / \operatorname{rad} R(G) \cong F(G) / \operatorname{rad} F(G)$, there is an $R(G)$-module $T$ such that

$$
W \cong T^{F} \cong V_{1} \oplus \cdots \oplus V_{t}
$$

Therefore $(m(D) T)^{K} \cong M$; and since $M$ is irreducible, $m(D)=1$. Since $N^{*}$ is realizable in $Z_{p}(\chi)$, it will be realizable in $K(\chi)$; so $\mathrm{m}_{K}(N)=1$. (d) and (e) are now immediate.

COROLLARY 1.5. The characters of the nonisomorphic irreducible $K(G)$-modules are linearly independent over $K$.

Proof. The characters of the nonisomorphic $E(G)$-modules are linearly independent over $E$ [3, Th. 30.12]. Since the characters of $M$ and $M^{E}$ are identical, the desired result is immediate from Theorem 1.4 (a) and (c).

REMARK. We have only stated the results concerning the Schur index that we will need in the following sections. Analogues of the other important theorems found in $[3, \S 70]$ can also be easily proved by the methods used here.

It will be useful to have an expression for the relationship between the simple component $A$ of $K(G) / \operatorname{rad} K(G)$ and the irreducible $E(G)$-module $N$.

Definition 1.6. Let $K$ be an arbitrary field, $E$ a finite separable extension of $K$. The simple component $A$ of $K(G) / \mathrm{rad} K(G)$ is associated with the irreducible $E(G)$-module $N$ if $N$ is isomorphic to a minimal left ideal of $A \otimes_{K} E$.

2. Outer tensor products of irreducible modules. Throughout this section $K$ will denote an arbitrary field, $G_{1}$ and $G_{2}$ will denote finite groups, and $G$ will be the direct product of $G_{1}$ and $G_{2}, G=G_{1} \times G_{2}$. 
$E$ will denote a finite normal separable extension of $K$ which is a splitting field for $G$. $M_{i}$ will be an irreducible $K\left(G_{i}\right)$-module, $i=1,2$, and $M_{1} \# M_{2}$ will denote the outer tensor product of $M_{1}$ and $M_{2} . A_{i}$ will denote the simple component of $K\left(G_{i}\right) / \mathrm{rad}\left(K G_{i}\right)$ corresponding (in the sense of Definition 1.6) to $M_{i}, i=1,2$. Let $N_{i}$ be an irreducible $E\left(G_{i}\right)$-component of $M_{i}^{E}$. For any $\sigma, \tau \in \mathscr{G}(E \mid K), \sigma N_{1} \# \tau N_{2}$ is an irreducible $E(G)$-module [1, Footnote, p. 587]. $\sigma N_{1} \# \tau N_{2}$ will not, in general, be a conjugate of $N_{1} \# N_{2}$. We shall let $\psi_{i}$ denote the character of $N_{i}, i=1,2$. All fields considered will be assumed to be subfields of $E^{*}$, a fixed algebraic closure of $E$. Let $L_{i}=K\left(\psi_{i}\right)$, $\mathscr{\mathscr { C } _ { i }}=\mathscr{C}\left(E \mid L_{i}\right), i=1,2$. Let $\overline{\mathscr{H}_{i}}$ be a fixed set of coset representatives of $\mathscr{H}_{i}$ in $\mathscr{G}(E \mid K)$. Theorem 1.4 (a) implies that $M_{i}^{E} \cong$ $m_{K}\left(N_{i}\right)\left(\sum \sigma_{i} N_{i}\right)$, the sum being over all $\sigma_{i} \in \overline{\mathscr{C}}_{i}$. Since the $\left\{L_{i}\right\}$ are normal over $K$, there is an unique composite $L$ of $L_{1}$ and $L_{2}$ over $K$.

Proposition 2.1. $m_{K}\left(N_{1} \# N_{2}\right)=m_{K}\left(\sigma N_{1} \# \tau N_{2}\right)$ for all $\sigma, \tau \in \mathscr{G}(E \mid K)$.

Proof. Let $\sigma, \tau \in \mathscr{G}(E \mid K)$. We may clearly assume that $\sigma \in \overline{\mathscr{H}}_{1}$, $\tau \in \overline{\mathscr{H}}_{2}$. In $\S 1$ we observed that $K\left(\psi_{i}\right)=K\left(\sigma \psi_{i}\right)=L_{i}, i=1$, 2, for all $\sigma \in \mathscr{G}(E \mid K)$. Let $L$ be the composite of $L_{1}$ and $L_{2}$ over $K$. Then

$$
m_{K}\left(N_{1} \# N_{2}\right)=m_{L}\left(N_{1} \# N_{2}\right), m_{K}\left(\sigma N_{1} \# \tau N_{2}\right)=m_{L}\left(\sigma N_{1} \# \tau N_{2}\right) \text {. }
$$

Because of Theorem 1.4 (c) we may assume that $K$ has characteristic zero. Let $C, D$ be the simple components of $L(G)$ associated with $N_{1} \# N_{2}$ and $\sigma N_{1} \# \tau N_{2}$ respectively. In view of Theorem 1.4 (b) it is sufficient to prove that $C \cong D$. Since $K(G) \cong K\left(G_{1}\right) \otimes_{K} K\left(G_{2}\right), A_{1} \otimes_{K} A_{2}$ is a component of $K(G)$. Therefore $A_{1}^{L} \otimes_{L} A_{2}^{L} \cong\left(A_{1} \otimes_{K} A_{2}\right)^{L}$ is a component of $L(G)$. It follows from Theorem 1.2 that $A_{1}^{L} \otimes_{L} A_{2}^{L}$ is a direct sum of isomorphic simple algebras. From Theorem 1.4 (a) we have $M_{1}^{E} \cong$ $m_{K}\left(N_{i}\right)\left(\sum \sigma_{i} N_{i}\right)$, the sum being over all $\sigma_{i} \in \overline{\mathscr{C}}_{i}$. Then

$$
\left(M_{1} \# M_{2}\right)^{E} \cong M_{1}^{E} \# M_{2}^{E} \cong m_{K}\left(N_{1}\right) m_{K}\left(N_{2}\right) \sum \alpha N_{1} \# \beta N_{2},
$$

where the sum is taken over all $\alpha \in \overline{\mathscr{H}}_{1}, \beta \in \overline{\mathscr{H}}_{2}$. From this we see that both $N_{1} \# N_{2}$ and $\sigma N_{1} \# \tau N_{2}$ are associated with $A_{1} \otimes_{K} A_{2}$, for all $\sigma \in \overline{\mathscr{H}_{1}}, \tau \in \overline{\mathscr{H}_{2}}$. This proves that $C$ and $D$ are components of $A_{1}^{L} \otimes_{L} A_{2}^{L}$ and so $C \cong D$.

Using this result we can determine the structure of $M_{1} \# M_{2}$ We first recall some properties of principal indecomposable modules [3, $\S 54]$. Let $V_{1}, V_{2}$ be principal indecomposable $K(G)$-modules. Then $V_{i} \cong K(G) e_{i}$ from some primitive idempotent $e_{i}, i=1,2$. $V_{i}$ has an unique maximal submodule isomorphic to $\operatorname{rad} K(G) e_{i}$. We denote this submodule $\operatorname{rad} V_{i} . \quad K(G) e_{i} / \operatorname{rad} K(G) e_{i}$ is an irreducible $K(G)$-module which we denote by $\bar{V}_{i}, i=1,2$. $V_{1} \cong V_{2}$ if and only if $\bar{V}_{1} \cong \bar{V}_{2}$. 
Theorem 2.2. $M_{1} \# M_{2}$ is completely reducible. $M_{1} \# M_{2} \cong$ $k\left(T_{1} \oplus \cdots \oplus T_{r}\right)$, where the $\left\{T_{i}\right\}$ are nonisomorphic irreducible $K(G)$ modules and $k=m_{K}\left(N_{1}\right) m_{K}\left(N_{2}\right) / m_{K}\left(N_{1} \# N_{2}\right)$. The $\left\{T_{\imath}\right\}$ have common $K$-dimension $s$, where $s=m_{K}\left(N_{1} \# N_{2}\right)(L: K)\left(N_{1} \# N_{2}: E\right)$.

Proof. Let $V_{i 1}, V_{i 2}, \cdots, V_{i n(i)}$ be the set of (isomorphic) principal indecomposable $K\left(G_{i}\right)$-modules such that $\bar{V}_{i j} \cong M_{i}, i=1,2, j=1,2, \cdots$, $n(i)$. Let $C_{i}=V_{i 1} \oplus V_{i 2} \oplus \cdots \oplus V_{i n(i)} . \quad C_{i}$ is a component of $K\left(G_{i}\right)$, $i=1,2 . \quad \bar{C}_{i}=\bar{V}_{i 1} \oplus \cdots \oplus \bar{V}_{i n(i)} \cong M_{i} \oplus \cdots \oplus M_{i}, i=1,2 . \quad \bar{C}_{i}$ is the sum of all the minimal left ideals of $K\left(G_{i}\right) / \operatorname{rad} K\left(G_{i}\right)$ which are isomorphic to $M_{i}, i=1,2$. Therefore $\bar{C}_{i}$ is a simple component of $K\left(G_{i}\right) / \mathrm{rad} K\left(G_{i}\right), i=1,2$ [3, Th. 25.15]. Let $N$ be the radical of $\bar{C}_{1} \otimes_{K} \bar{C}_{2}$. Then $N^{L}$ is contained in the radical of $\bar{C}_{1}^{L} \otimes_{L} \bar{C}_{2}^{L}$. But $\bar{C}_{1}^{L} \cong D_{i 1} \oplus \cdots \oplus D_{i m(i)}, i=1,2$, where the $\left\{D_{i j}\right\}$ are central simple algebras over $L$. Therefore $\bar{C}_{1}^{L} \otimes_{L} \bar{C}_{2}^{L}$ has zero radical, so $N=0$, i.e. $\bar{C}_{1} \otimes_{K} \bar{C}_{2} \cong C_{1} \otimes C_{2} / \mathrm{rad}\left(C_{1} \otimes_{K} C_{2}\right)$. Since $C_{1} \otimes_{K} C_{2}$ is a component of $K(G)$, we may express it as a direct sum of principal indecomposable $K(G)$-modules, $C_{1} \otimes_{K} C_{2}=Y_{1} \oplus \cdots \oplus Y_{s}$. Then $C_{1} \otimes_{K} C_{2} / \operatorname{rad} C_{1} \otimes_{K} C_{2} \cong$ $\bar{Y}_{1} \oplus \cdots \oplus \bar{Y}_{s}$ and the $\left\{\bar{Y}_{i}\right\}$ are irreducible $K(G)$-modules. We have

$$
\bar{Y}_{1} \oplus \cdots \oplus \bar{Y}_{s} \cong \bar{C}_{1} \otimes_{K} \bar{C}_{2} \cong \sum_{i, j} \bar{V}_{1 i} \otimes_{K} \bar{V}_{2 j} \cong \sum M_{1} \# M_{2} .
$$

Let $M_{1} \# M_{2}=X_{1} \oplus \cdots \oplus X_{r}$, the $X_{i}$ being indecomposable $K(G)$ modules. By the Krull-Schmidt Theorem each $X_{i}$ is isomorphic to some $\bar{Y}_{\jmath}$. Therefore $M_{1} \# M_{2}$ is completely reducible [3, Th. 15.3]. Let $M_{1} \# M_{2} \cong \bar{Y}_{1} \oplus \cdots \oplus \bar{Y}_{r}$. We have previously observed that

$$
\left(M_{1} \# M_{2}\right)^{E} \cong m_{K}\left(N_{1}\right) m_{K}\left(N_{2}\right)\left(\sum \sigma N_{1} \# \tau N_{2}\right), \sigma \in \overline{\mathscr{C}_{1}}, \tau \in \overline{\mathscr{C}}_{2} .
$$

Let $\sigma_{1}, \sigma_{2}$ be elements of $\overline{\mathscr{Z} \mathscr{C}_{1}}$. If $\sigma_{1} N_{1} \# \tau_{1} N_{2} \cong \sigma_{2} N_{2} \# \tau_{2} N_{2}$ where $\tau_{1}$ and $\tau_{2}$ are in $\overline{\mathscr{C}} \mathscr{C}_{2}$, then $\sigma_{1} \psi_{1}=\sigma_{2} \psi_{2}$ and so $\sigma_{1}=\sigma_{2}$. Since $\left(M_{1} \# M_{2}\right)^{E} \cong$ $Y_{1}^{E} \oplus \cdots \oplus Y_{r}^{E}$, it is immediate from Proposition 2.1 that $M_{1} \# M_{2} \cong$ $k\left(T_{1} \oplus \cdots \oplus T_{r}\right)$, where the $T_{i}$ are nonisomorphic irreducible $K(G)$ modules and $k=m_{K}\left(N_{2}\right) / m_{K}\left(N_{1} \# N_{2}\right)$. The $K$-dimension of any of the $T_{i}^{\prime} \mathrm{S}$ is $\mathrm{m}_{K}\left(N_{1} \# N_{2}\right)(L: K)\left(N_{1} \# N_{2}: E\right)$ in view of Theorem 1.4 (a) and $K\left(\psi_{1}, \psi_{2}\right)=K\left(\sigma \psi_{1}, \tau \psi_{2}\right)=L$ for all $\sigma, \tau \in \mathscr{G}(E \mid K)$.

REMARK. Let $V_{i}$ be a principal indecomposable $K\left(G_{i}\right)$-module $i=1$, 2. The proof of Theorem 2.2 shows that $V_{1} \# V_{2}$ is a principal indecomposable $K(G)$-module if and only if $\bar{V}_{1} \# \bar{V}_{2}$ is an irreducible $K(G)$-module. If the $V_{i}$ are indecomposable, but not necessarily principal indecomposable $K\left(G_{i}\right)$-modules, $i=1,2$, then $V_{1} \# V_{2}$ is indecomposable if and only if $d_{G_{1}}\left(V_{1}\right) \otimes_{K} d_{G_{2}}\left(V_{2}\right)$ is a division algebra, where

$$
d_{G_{i}}\left(V_{i}\right)=\underset{K\left(G_{i}\right)}{\operatorname{Hom}}\left(V_{i}, V_{i}\right) / \operatorname{rad} \underset{K\left(G_{i}\right)}{\operatorname{Hom}}\left(V_{1}, V_{i}\right), \quad i=1,2
$$


$[5$, p. 438].

We now turn to the question of when $M_{1} \# M_{2}$ is irreducible.

THEOREM 2.3. $\quad M_{1} \# M_{2}$ is an irreducible $K(G)$-module if and only if the following conditions are satisfied:

(a) $m_{K}\left(N_{1}\right) m_{K}\left(N_{2}\right)=m_{K}\left(N_{1} \# N_{2}\right)$.

(b) $\mathscr{G}(E \mid K)=\mathscr{H}_{1} \mathscr{H}_{2}$.

(c) $\left(K\left(\psi_{1}\right): K\right) \cdot\left(K\left(\psi_{2}\right): K\right)=\left(K\left(\psi_{1}, \psi_{2}\right): K\right)$.

Proof. We begin by showing that $\mathscr{G}(E \mid K)=\mathscr{H}_{1} \mathscr{K}_{2}$ if and only if $\sigma \mathscr{H}_{1} \cap \tau \mathscr{H}_{2}$ is nonempty for all $\sigma, \tau \in \mathscr{O}(E \mid K)$. Since $K\left(\psi_{i}\right)$ is a normal extension of $K, \mathscr{H}_{1}$ is a normal subgroup of $\mathscr{G}(E \mid K), i=$ 1,2. Assume that $\mathscr{G}(E \mid K)=\mathscr{C}_{1} \mathscr{C}_{2}$ and let $\sigma, \tau \in \mathscr{G}(E \mid K)$. Then $\sigma \mathscr{H}_{1}=h_{2} \mathscr{C}_{1}, \tau \mathscr{C}_{2}=h_{1} \mathscr{C}_{2}$ where $h_{i} \in \mathscr{C}_{i}, i=1,2$. Then

$$
h_{2} h_{1} \in \sigma \mathscr{H}_{1} \cap \tau \mathscr{H}_{2}=h_{2} \mathscr{H}_{1} \cap \mathscr{H}_{2} h_{1} .
$$

Conversely, assume that $\sigma \mathscr{H}_{1} \cap \tau \mathscr{H}_{2}$ is nonempty for all $\sigma, \tau \in \mathscr{P}(E \mid K)$ and let $x \in \mathscr{G}(E \mid K)$. Then $x \mathscr{C}_{1} \cap \mathscr{H}_{2}$ is nonempty so $x h_{1}=h_{2}$ for some $h_{1} \in \mathscr{H}_{1}, h_{2} \in \mathscr{C}_{2}$. Therefore $x \in \mathscr{H}_{2} \mathscr{C}_{1}=\mathscr{H}_{1} \mathscr{K}_{2}$ so $\mathscr{C}(E \mid K)=$ $\mathscr{C}_{1} \mathscr{C}_{2}$. We have $\left(M_{1} \# M_{2}\right)^{E} \cong m_{K}\left(N_{1}\right) m_{K}\left(N_{2}\right)\left(\sum \sigma N_{1} \approx N_{2}\right)$, the sum take over all $\sigma \in \overline{\mathscr{C}}_{1}, \tau \in \overline{\mathscr{C}}_{2}$. Assume that $M_{1} \# M_{2}$ is irreducible. By Theorem 1.4 (a) we see that (a) is necessary. For each $\sigma \in \mathscr{H}_{1}, \tau \in \mathscr{C}_{2}$ there must exist a $\gamma \in \mathscr{G}(E \mid K)$ such that $\sigma N_{1} \# \tau N_{2} \cong \gamma N_{1} \# \gamma N_{2} \cong$ $\gamma\left(N_{1} \# N_{2}\right)$. Then $\lambda \in \sigma \mathscr{H}_{1} \cap \tau \mathscr{H}_{2}$ so (b) is necessary. By Theorem 1.4 (a) the total number of composition factors of $\left(M_{1} \# M_{2}\right)^{E}$ must be $m_{K}\left(N_{1} \# N_{2}\right) \cdot\left(K\left(\psi_{1}, \psi_{2}\right): K\right)$. Therefore

$$
\left(K\left(\psi_{1}, \psi_{2}\right): K\right)=\left(K\left(\psi_{1}\right): K\right) \cdot\left(K\left(\psi_{2}\right): K\right)
$$

so (c) is necessary. The same argument shows that (a), (b) and (c) are sufficient since $\left(M_{1} \# M_{2}\right)^{E} \cong W^{E}, \quad W$ an irreducible $K(G)$-module, implies $M_{1} \# M_{2} \cong W$.

Corollary 2.4. Let $G_{1}=G_{2}, G=G_{1} \times G_{1}$. Let $M_{1}$ be an irreducible $K\left(G_{1}\right)$-module. Then $M_{1} \# M_{1}$ is irreducible if and only if $M_{1}$ is an absolutely irreducible $K\left(G_{1}\right)$-module.

Proof. The if part of the theorem is immediate from [1, Footnote, p. 587]. Conversely, let $M_{1} \# M_{1}$ be irreducible. In order for condition (b) of Theorem 2.3 hold, $K\left(\psi_{1}\right)$ must equal $K$. If $N_{1}^{*}$ is realizable (in the context of $\S 1$ ) in a field $F$ then so is $N_{1}$. Therefore $m_{K}\left(N_{1} \# N_{1}\right) \leqq m_{K}\left(N_{1}\right)$. But then condition (a) of Theorem 2.3 holds if and only if $m_{K}\left(N_{1}\right)=1$. Therefore $N_{1}^{*}$ is realizable in $K$ and so $M_{1}$ is absolutely irreducible. 
The next result gives a more easily applied criterion for $M_{1} \# M_{2}$ to be irreducible.

THEOREM 2.5. Let $K\left(\psi_{1}\right)=K$ and assume that $\left(m_{K}\left(N_{1}\right), m_{K}\left(N_{2}\right)\right)=$ 1. Then $M_{1} \# M_{2}$ is irreducible.

Proof. Since $K\left(\psi_{1}\right)=K$, conditions (b) and (c) of Thereom 2.3 are satisfied so we need only prove that $m_{K}\left(N_{1} \# N_{2}\right)=m_{K}\left(N_{1}\right) m_{K}\left(N_{2}\right)$. It follows from Theorem 2.2 that $m_{K}\left(N_{1}\right) m_{K}\left(N_{2}\right) \geqq m_{K}\left(N_{1} \# N_{2}\right)$. Since $\left(m_{K}\left(N_{1}\right), m_{K}\left(N_{2}\right)\right)=1$ we need only show that both $m_{K}\left(N_{1}\right)$ and $m_{K}\left(N_{2}\right)$ divide $m_{K}\left(N_{1} \# N_{2}\right)$. By Theorem 1.4 (c) we may assume that $K$ has characteristic zero. Let $F$ be a maximal subfield of the division algebra component of $A_{1} \otimes_{K} A_{2}$. Then $N_{1}^{*} \# N_{2}^{*}$ is realizable in $F$ and $\left(F: K\left(\psi_{1}, \psi_{2}\right)\right)=m_{K}\left(N_{1} \# N_{2}\right)$ [3, Th. 68.6]. In view of Theorem 1.4 (e) it is sufficient to prove that $N_{1}^{*}$ and $N_{2}^{*}$ are realizable in $F$. Let $B_{i}$ be the simple component of $A_{i} \otimes_{K} F$ corresponding to $N_{i}^{*}$. Since $N_{1}^{*} \# N_{2}^{*}$ is realizable in $F, B_{1} \bigotimes_{F} B_{2} \cong(F)_{r}$. Therefore $B_{1}$ and $B_{2}$ are inverse isomorphic elements of the Brauer group of $F$ and hence their division algebra components have the same index. If $B_{i}=\left(D_{i}\right)_{n(i)}$ we have $m\left(D_{1}\right)=m\left(D_{2}\right)$. From Theorem 1.4 (c) we have $m_{F}\left(N_{1}^{*}\right)=$ $m_{F}\left(N_{2}^{*}\right)$. Since $m_{F}\left(N_{1}^{*}\right)$ divides $m_{K}\left(N_{1}\right)$ and $\left(m_{K}\left(N_{1}\right), m_{K}\left(N_{2}\right)\right)=1$, $m_{F}\left(N_{1}^{*}\right)=m_{F}\left(N_{2}^{*}\right)=1$.

COROLlaRY 2.6. Let the orders of $G_{1}$ and $G_{2}$ be relatively prime. If $K\left(\psi_{1}\right)=K$, then $M_{1} \# M_{2}$ is irreducible.

Proof. By Theorem 1.4 (d) $m_{K}\left(N_{i}\right)$ divides $\left(N_{i}: E\right), i=1,2$. For $K$ of characteristic zero, $\left(N_{i}: E\right)$ divides the order of $G_{i}$ [3, Th. 33.7]. For $K$ of characteristic $p$ we have $m_{K}\left(N_{i}\right)=1, i=1,2$. In both cases Corollary 2.6 is immediate from the preceding theorem.

Given an irreducible $K(G)$-module $M$, it is natural to ask when $M$ is isomorphic to $M_{1} \# M_{2}$ for irreducible $K\left(G_{i}\right)$-modules $M_{i}, i=1,2$. If such $M_{i}$ exist, $i=1,2$, we say that $M$ is factorizable. If $M$ is a $K(G)$-module, we denote by $M_{G_{i}}$ the left $K\left(G_{i}\right)$-module obtained by restriction of the set of operators on $M$ from $K(G)$ to $K\left(G_{i}\right), i=1,2$.

THEOREM 2.7. Let $M$ be an irreducible $K(G)$-module. Then $M_{G_{i}} \cong e_{i} M_{i}$ for irreducible $K\left(G_{i}\right)$-modules $M_{i}, i=1,2$. $M$ is factorizable if and only if $M_{1} \# M_{2}$ is irreducible, in which case $M \cong M_{1} \# M_{2}$.

Proof. $M_{G_{i}} \cong e_{i}\left(M_{i} \oplus M_{i}^{(g)} \oplus \cdots \oplus M_{i}^{(h)}\right)$, where the $M_{i}^{(g)}$ are conjugates by elements of $G$ of the irreducible $K\left(G_{i}\right)$-module $M_{i}, i=1,2$ [3, Th. 49.2]. Since $G_{1}$ and $G_{2}$ commute, all conjugates of $M_{i}$ are 
equivalent so $M_{G_{i}} \cong e_{i} M_{i}$, $i=1,2$. Let

$$
M^{E} \cong m_{K}(N)\left(\sum_{\jmath} \sigma_{\jmath} N\right), \sigma_{j} \in \mathscr{G}(E \mid K),
$$

where $N$ is an irreducible $E(G)$-module. Since $N$ is factorizable, we have $N \cong N_{1} \# N_{2}$ where the $N_{i}$ are irreducible $E\left(G_{i}\right)$-modules, $i=1,2$. Then $M^{E} \cong m_{K}(N)\left(\sum_{j} \sigma_{j}\left(N_{1} \# N_{2}\right)\right.$. Since

$$
N_{G_{i}} \cong f_{i} N_{i},\left(M^{E}\right)_{G_{i}} \cong f_{i} m_{K}(N)\left(\sum_{j} \sigma_{j} N_{i}\right)
$$

so $\left(M_{\theta_{i}}\right)^{E} \cong\left(M^{E}\right)_{G_{i}} \cong f_{i} m_{K}(N)\left(\sum_{j} \sigma_{j} N_{i}\right)$. Therefore

$$
\left(M_{i}\right)^{E} \cong \frac{f_{i} m_{K}(N)}{e_{i}}\left(\sum_{j} \sigma_{\jmath} N_{i}\right)
$$

so $N_{1} \# N_{2}$ is a component of both $M^{E}$ and $\left(M_{1} \# M_{2}\right)^{E}$. If $M \cong$ $M_{1}^{\prime} \# M_{2}^{\prime},\left\{M_{i}^{\prime}\right\}$ irreducible $K\left(G_{i}\right)$-modules, then $M_{\theta_{i}} \cong k_{i} M_{i}^{\prime}$; and so $\left(M_{i}^{\prime}\right)^{E}$ and $\left(M_{i}\right)^{E}$ both have $N_{i}$ as a component, $i=1,2$. Therefore $M_{i} \cong M_{\imath}^{\prime}, i=1,2$; and similarly, if $M_{1} \# M_{2}$ is irreducible, we have $M \cong M_{1} \# M_{2}$ 。

The well known theory of central simple algebras over algebraic number fields has an interesting application to outer tensor products. Let $K$ be an algebraic number field, $G$ an arbitrary finite group, and $E$ a finite normal extension of $K$ which is a splitting field for $G$. We denote by $G^{(r)}$ the direct product of $G$ with itself $r$ times. Let $N$ be an irreducible $E(G)$-module. $N^{(r)}$ will denote the $E\left(G^{(r)}\right)$-module $N \# N \# \cdots \# N$, the outer tensor product of $N$ with itself $r$ times. Let $\psi$ be the character of $N$.

THEOREM 2.8. $m_{K}(N)$ is the smallest integer $r$ such that $N^{(r)}$ is realizable in $K(\psi)$.

Proof. Since $m_{K}(N)=m_{K(\psi)}(N)$ we may assume that $K(\psi)=K$. Let $A$ be the simple component of $K(G)$ corresponding to $N$. Then $A^{(r)}=A \otimes_{K} A \otimes_{K} \cdots \otimes_{K} \cdots \otimes_{K} A, r$ times, is the simple component of $K\left(G^{(r)}\right)$ corresponding to $N^{(r)}$. $N^{(r)}$ is realizable in $K$ if and only if $A^{(r)} \cong(K)_{s}$. Let $t$ be the exponent of $A$. Then $A^{(r)} \cong(K)_{s}$ if and only if $t$ divides $r$. Let $D$ be the division algebra component of $A$. Since $A$ is central simple over an algebraic number field, $t=m(D)$ [4, Satz 7, p. 119]. The desired conclusion now follows from Theorem $1.4(b)$.

3. Derivable division algebras. Let $D$ be a division algebra and $K$ a subfield of the center of $D$. 
Definition 3.1. $D$ is $K$-derivable if $D \cong \operatorname{Hom}_{K(G)}(M, M)$ for some finite group $G$ and irreducible $K(G)$-module $M$.

Theorems 2.3 and 2.5 have an immediate application to the theory of derivable division algebras.

Theorem 3.2. Let $D_{1}$ and $D_{2}$ be K-derivable division algebras. Let $L_{1}, L_{2}$ be the centers of $D_{1}$ and $D_{2}$ respectively. If $D_{1}$ is central over $K$, i.e. $L_{1}=K$, and if $\left(m\left(D_{1}\right), m\left(D_{2}\right)\right)=1$, then $D_{1} \otimes_{K} D_{2}$ is a division algebra. In general, the following conditions are necessary and sufficient for $D_{1} \otimes_{K} D_{2}$ to be a division algebra:

(a) $m\left(D_{1}\right) m\left(D_{2}\right)=m\left(D_{3}\right)$, where $D_{3}$ is the division algebra component of a simple component of $\left(D_{1} \otimes_{K} D_{2}\right) \bigotimes_{K} L_{1} \cdot L_{2}, L_{1} \cdot L_{2}$ being a composite of $L_{1}$ and $L_{2}$ over $K$.

(b) Let $E$ be a finite normal extension of $K$ which is a splitting field for $D_{1}$ and $D_{2}$. Then $\mathscr{G}(E \mid K)=\mathscr{C}_{1} \mathscr{Y}_{2}$ where $\mathscr{Y}_{i}=$ $\mathscr{G}\left(E \mid L_{i}\right), i=1,2$.

(c) $\left(L_{1}: K\right)\left(L_{2}: K\right)=\left(L_{1} \cdot L_{2}: K\right)$.

Proof. Let $D_{i} \cong \operatorname{Hom}_{K\left(G_{i}\right)}\left(M_{i}, M_{i}\right), \quad i=1,2$. Set $G=G_{1} \times G_{2}$. Then

$$
\underset{K(\alpha)}{\operatorname{Hom}}\left(M_{1} \# M_{2}, M, \# M_{2}\right) \cong \underset{K\left(G_{1}\right)}{\operatorname{Hom}}\left(M_{1}, M_{1}\right) \underset{K}{\bigotimes_{K\left(G_{2}\right)}} \underset{\operatorname{Hom}}{ }\left(M_{2}, M_{2}\right) \cong D_{1} \bigotimes_{K} D_{2} .
$$

If $M_{1} \# M_{2}$ is an irreducible $K(G)$-module, then $D_{1} \otimes_{K} D_{2}$ is a skewfield [3, Th. 26.8]. Conversely, if $D_{1} \otimes_{K} D_{2}$ is a skewfield, then $M_{1} \# M_{2}$ is indecomposable. By Theorem 2.2 $M_{1} \# M_{2}$ is irreducible. Theorems 2.3 and 2.5 now yield the desired result.

If $K$ is an infinite field of prime characteristic, there will, in general, exist division algebras central over $K$ which are not fields. Theorem 1.4 (c) proves that such division algebras are not derivable. We now consider fields of characteristic zero. If $D$ is a $K$-derivable division algebra and $L$ is the center of $D$, then Theorem 1.1 shows that $D$ is $L$-derivable. For this reason we shall consider only central division algebras. Our final result shows the existence of infinitely many division algebras $D$ which are not $K$-derivable for any subfield $K$ of $D$.

Let $B(K)$ denote the Brauer group of $K$. Let $B_{0}(K)$ be the subset of $B(K)$ consisting of those classes of central simple algebras which have $K$-derivable division algebra components.

Theorem 3.3. $B_{0}(K)$ is a subgroup of $B(K)$. If $K$ is an algebraic number field which is not an abelian extension of the rationals, then $B_{0}(K)$ has infinite index in $B(K)$.

Proof. $K$ is $K$-derivable since $K \cong \operatorname{Hom}_{K(\theta)}(N, N)$ with $G$ the 
identity group and $N$ the trivial $K(G)$-module. Therefore $B_{0}(K)$ is nonempty. Since every element of $B(K)$ has finite order, to show that $B_{0}(K)$ is a subgroup of $B(K)$ it is sufficient to prove that $B_{0}(K)$ is closed under $\bigotimes_{K}$. Let $\left\{A_{1}\right\},\left\{A_{2}\right\} \in B_{0}(K)$ with $D_{i} \in\left\{A_{i}\right\}, D_{i}$ a division algebra central over $K$ and $D_{i} \cong \operatorname{Hom}_{K\left(\theta_{i}\right)}\left(M_{i}, M_{i}\right), i=1,2$. Let $G=$ $G_{1} \times G_{2}$. Then $D_{1} \otimes_{K} D_{2} \cong \operatorname{Hom}_{K(G)}\left(M_{1} \# M_{2}, M_{1} \# M_{2}\right)$. From the proof of Theorem 2.2 we see that $M_{1} \# M_{2} \cong k N, N$ an irreducible $K(G)$ module. Let $\operatorname{Hom}_{K^{\prime}(G)}(N, N)=D_{3}, D_{3}$ a division algebra central over $K$. Then $\operatorname{Hom}_{K(G)}\left(M_{1} \# M_{2}, M_{1} \# M_{2}\right) \cong\left(D_{3}\right)_{k}$ so $D_{1} \bigotimes_{K} D_{2}=\left(D_{3}\right)_{r}$. Therefore $A_{1} \otimes_{K} A_{2} \cong\left(D_{3}\right)_{s}$, so $B_{0}(K)$ is a subgroup of $B(K)$.

Assume that $K$ is an algebraic number field which is not an Abelian extension of the rationals. Let $L$ be the maximal abelian subfield of $K$. There exists a rational prime $p$ which splits completely in $K$ [6, Satz 114, p. 126]. (As Dr. Basil Gordon has pointed out, this result can also be proved purely algebraically.) Let $L_{0}, \vec{\Lambda}_{0}$ denote the rings of algebraic integers of $L, K$, respectively. There exist prime ideals $\mathfrak{Y}_{1}$ and $\mathfrak{Y}_{2}$ of $K_{0}$ such that $\mathfrak{Y}_{1} \cap L_{0}=\mathfrak{Y}_{2} \cap L_{0}$ and $\mathfrak{Y}_{1} \cap Z=$ $\mathfrak{Y}_{2} \cap Z=(p), Z$ the ring of rational integers [9, Corollary, p. 287]. There exists a division algebra $D$ central over $K$ for which $h\left(D, \mathfrak{Y}_{1}\right)=$ $1 / 3, h\left(D, \mathfrak{Y}_{2}\right)=2 / 3, h(D, \mathfrak{Y})=0$ for all other primes $\mathfrak{Y}$ of $K$, finite or infinite, where $h(D, \mathfrak{Y})$ denotes the Hasse invariant of $D$ at $\mathfrak{Y}$ [4, Satz 9, p. 119]. We shall show that $D$ is not $K$-derivable.

Suppose $D \cong \operatorname{Hom}_{K(G)}(M, M)$ for some finite group $G$ and irreducible $K(G)$-module $M$. Let $E$ be a finite normal extension of $K$ which is a splitting field for $G$. Since $D$ is central over $K, M^{E}=m_{K}(N) \cdot N$, $N$ an irreducible $E(G)$-module. Let $\psi$ be the character of $N$. Let $Q$ be the field of rational numbers. Then $Q(\psi)$ is a subfield of $K$ and since $Q(\psi)$ is an Abelian extension of $Q, Q(\psi)$ is a subfield of $L$. Let $A$ be the simple component of $Q(\psi)(G)$ corresponding to $N$. Then $A$ is a central simple $Q(\psi)$-algebra, and $A \otimes_{\mathcal{Q}(r)} K \cong(D)_{r}$. Since $\mathfrak{Y}_{1} \cap L_{0}=$ $\mathfrak{Y}_{2} \cap L_{0}$ we have $\mathfrak{Y}_{1} \cap Q(\psi)_{0}=\mathfrak{Y}_{2} \cap Q(\psi)_{0}=\mathfrak{Y}_{3}$. Let $n\left(\mathfrak{Y}_{i}\right)$ denote the residue class degree of $\mathfrak{Y}_{i}$ over $\mathfrak{Y}_{3}, i=1,2$. Since $p$ splits completely in $K, n\left(\mathfrak{Y}_{1}\right)=n\left(\mathfrak{Y}_{2}\right)=1$. Let $h\left(A, \mathfrak{Y}_{3}\right)$ be the Hasse invariant of $A$ at $\mathfrak{Y}_{3}$. Then $n\left(\mathfrak{Y}_{i}\right) h\left(A, \mathfrak{Y}_{3}\right)=h\left(A, \mathfrak{Y}_{3}\right)$ is the Hasse invariant of $D$ at $\mathfrak{Y}_{i}$, $i=1,2[4$, Satz $4, p, 113]$. This contradicts the fact that $h(D, \mathfrak{Y})_{1} \neq$ $h\left(D, \mathfrak{Y}_{2}\right)$.

We have shown that $B_{0}(K)$ is a proper subgroup of $B(K)$. Suppose that $B_{0}(K)$ has finite index $n$ in $B(K)$. Let $\mathfrak{Y}_{+}$be a prime ideal of $K_{0}, \mathfrak{Y}_{4}$ distinct from both $\mathfrak{Y}_{1}$ and $\mathfrak{Y}_{2}$. Let $\left\{A_{1}\right\}$ be the class of central simple $K$-algebras whose Hasse invariants are

$$
h\left(A_{1}, \mathfrak{Y}_{1}\right)=1 / 3 n, h\left(A_{1}, \mathfrak{Y}_{2}\right)=2 / 3 n, h\left(A_{1}, \mathfrak{Y}_{\sharp}\right)=(n-1) / n, h\left(A_{1}, \mathfrak{Y}\right)=0
$$

for all other primes of $K$, finite or infinite. Let $\left\{A_{1}\right\}^{n}$ be the $n$th 
power of $\left\{A_{1}\right\}$ in $B(K)$ i.e. $\left.\left\{A_{1}\right\}^{n}=A_{1} \otimes_{K} A_{1} \otimes_{K} \cdots \otimes_{K} A_{1}\right\}$. The Hasse invariants of $\left\{A_{1}\right\}^{n}$ are precisely the Hasse invariants of $\{D\}[4$, Satz 3, p. 112]. Therefore $\left\{A_{1}\right\}^{n}$ equals $\{D\}$ [4, Satz 8, p. 119]. But $B_{0}(K)$ has index $n$ in $B(K)$ so $\left\{A_{1}\right\}^{n} \in B_{0}(K)$. This contradicts $\{D\} \notin B_{0}(K)$, so $B_{0}(K)$ is a subgroup of infinite index in $B(K)$.

\section{BIBLIOGRAPHY}

1. R. Brauer and C. Nesbitt, On the modular characters of groups, Ann. of Math. 42 (1941), 556-590.

2. R. Brauer and E. Noether, Über minimale Zerfällungskörper irreduzibler Darstellungen, S.-B. preuss Akad. Wiss. 32 (1927), 221-226.

3. C. W. Curtis and I. Reiner, Representation Theory of Finite Groups and Associative Algebras, Interscience, New York, 1962.

4. M. Deuring, Algebren, Springer, Berlin, 1935.

5. J. A. Green, On the indecomposable representations of a finite group, Math. Zeit. 70 (1959), 430-445.

6. E. Hecke, Vorlesungen uber die Theorie der algebraischen Zahlen, Akademische Verlag, Leipzig, 1923.

7. N. Jacobson, The Structure of Rings, Amer. Math. Soc., Providence, 1956.

8. - Lectures in Abstract Algebra, Vol. III, Van Nostrand, Princeton, 1964.

9. O. Zariski and P. Samuel, Commutative Algebra, Vol. 1, Van Nostrand, Princeton, 1958.

Received October 7, 1965.

University of CALifornia, Los ANGELeS 


\section{PACIFIC JOURNAL OF MATHEMATICS}

H. SAMELSON

Stanford University

Stanford, California

J. P. JaNs

University of Washington

Seattle, Washington 98105

\section{EDITORS}

J. DugundJI

University of Southern California Los Angeles, California 90007

RICHARD ARENS

University of California

Los Angeles, California 90024

\section{ASSOCIATE EDITORS}

E. F. BECKENBACH

B. H. NEUMANN

F. WOLF

K. YosidA

\section{SUPPORTING INSTITUTIONS}

UNIVERSITY OF BRITISH COLUMBIA

CALIFORNIA INSTITUTE OF TECHNOLOGY

UNIVERSITY OF CALIFORNIA

MONTANA STATE UNIVERSITY

UNIVERSITY OF NEVADA

NEW MEXICO STATE UNIVERSITY

OREGON STATE UNIVERSITY

UNIVERSITY OF OREGON

OSAKA UNIVERSITY

UNIVERSITY OF SOUTHERN CALIFORNIA
STANFORD UNIVERSITY

UNIVERSITY OF TOKYO

UNIVERSITY OF UTAH

WASHINGTON STATE UNIVERSITY

UNIVERSITY OF WASHINGTON

AMERICAN MATHEMATICAL SOCIETY CHEVRON RESEARCH CORPORATION TRW SYSTEMS

NAVAL ORDNANCE TEST STATION 


\section{Pacific Journal of Mathematics}

\section{Vol. 20, No. $1 \quad$ September, 1967}

Leonard Daniel Baumert, Extreme copositive quadratic forms. II ........ 1

Edward Lee Bethel, A note on continuous collections of disjoint

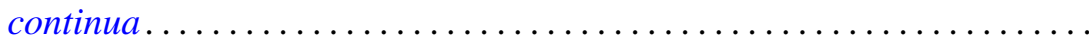

Delmar L. Boyer and Adolf G. Mader, A representation theorem for abelian groups with no elements of infinite p-height ...................

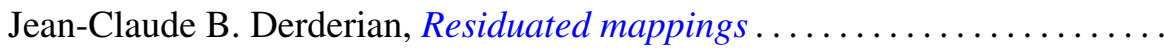

Burton I. Fein, Representations of direct products of finite groups ......... 45

John Brady Garnett, A topological characterization of Gleason parts.......

Herbert Meyer Kamowitz, On operators whose spectrum lies on a circle or

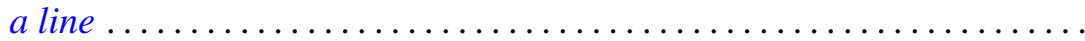

Ignacy I. Kotlarski, On characterizing the gamma and the normal distribution ........................................

Yu-Lee Lee, Topologies with the same class of homeomorphisms ..........

Moshe Mangad, Asymptotic expansions of Fourier transforms and discrete polyharmonic Green's functions ...........................

Jürg Thomas Marti, On integro-differential equations in Banach spaces ....

Walter Philipp, Some metrical theorems in number theory.............. 109

Maxwell Alexander Rosenlicht, Another proof of a theorem on rational

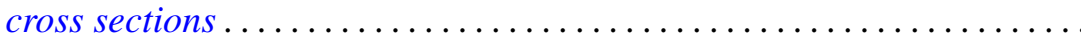

Kenneth Allen Ross and Karl Robert Stromberg, Jessen's theorem on Riemann sums for locally compact groups

Stephen Simons, A theorem on lattice ordered groups, results of Ptak, Namioka and Banach, and a front-ended proof of Lebesgue's theorem...

Morton Lincoln Slater, On the equation $\varphi(x)=\int_{x} x+1 K(\xi) f[\varphi(\xi)] d \xi \ldots$ 155 Arthur William John Stoddart, Existence of optimal controls .. 167 Burnett Roland Toskey, A system of canonical forms for rings on a direct sum of two infinite cyclic groups ....................

Jerry Eugene Vaughan, A modification of Morita's characterization of dimension 